\title{
Evaluation of Calretinin as a New Marker in the Diagnosis of Hirschsprung Disease
}

\author{
Mohammad Hossein Anbardar ${ }^{1}$; Bita Geramizadeh ${ }^{1,}$; Hamid Reza Foroutan $^{1}$ \\ ${ }^{1}$ Department of Pathology, Transplant Research Center, Shiraz University of Medical Sciences, Shiraz, IR Iran \\ *Corresponding author: Bita Geramizadeh, Department of Pathology, Transplant Research Center, Shiraz University of Medical Sciences, P. O. Box: 71345-1864, Shiraz, IR Iran. \\ Tel/Fax:+98-7116474331, E-mail: geramib@sums.ac.ir
}

Received: February 20, 2015; Revised: March 5, 2015; Accepted: March 22, 2015

\begin{abstract}
Background: Hirschsprung's disease (HD) is a congenital intestinal motility disorder with absence of ganglion cells in the colonic wall. Diagnosis of the disease is mainly based on the identification of the lack of ganglion cells in the pathology sections of the colon which is very difficult and time consuming and also needs several serial cut sections. There are many proposed markers in this field in the literature but none of them has been satisfactory. Calretinin immunohistochemistry (IHC) has been introduced as a new diagnostic marker to overcome the problems in diagnosis of this disease about 5 years ago. However there are few studies regarding the benefits and pitfalls of this marker.

Objectives: The aim of this study is to determine the diagnostic value of calretinin IHC in detecting aganglionosis(HD).

Patients and Methods: 27 HD patients and 28 non-Hirschsprung's disease (NHD) patients were collected in a prospective study and calretinin IHC was performed on 31 aganglionic and 51 normoganglionic full wall thickness sections of colectomies (some of the cases had more than 1 section). The IHC slides were evaluated by two pathologists and the diagnostic value was calculated in comparison with gold standard which is the presence or absence of ganglion cells in serial Hematoxylin and Eosin(HE) stained sections of the colectomies. Results: There was great concordance between the final diagnosis of both pathologists and gold standard (k $>0.9)$. Calretinin immunostaining showed $100 \%$ specificity and positive predictive value and more than $90 \%$ sensitivity and negative predictive value. High agreement was present between the two pathologists $(\mathrm{k}>0.9)$.

Conclusions: Calretinin IHC is a very convenient, useful and valuable method to demonstrate aganglionosis in HD patients. Loss of calretinin immunostaining in lamina propria and submucosa is characteristic of HD.
\end{abstract}

Keywords: Hirschsprung Disease; Immunohistochemistry; Ganglion Cells

\section{Background}

Hirschsprung's disease (HD) also known as congenital aganglionosis megacolon is a common malformation of enteric nervous system characterized by lack of ganglion cells in the submucosal (Meissner) and myenteric (Aurbach) nerve plexuses of distal colonic wall (1-4). HD affects 1 in 5000 liveborns and it is considered in differential diagnoses of patients with severe constipation in any age especially pediatric age group (5). The etiology of HD is not clearly described but thought to be related to abnormalities in craniocaudal migration of ganglion cells during the development of the intestine $(1,2)$. The diagnosis is based on clinical data, imaging studies and histological features together. Before surgery rectal biopsy has the pivotal role for the diagnosis of $\operatorname{HD}(2,3,5)$. However finding the ganglion cells or proof of the absence of ganglion cells needs numerous serial cut sections and is very time consuming and also has many pitfalls, therefore a number of ancillary methods like acetyl cholinesterase (AchE) histochemistry and various immunohistochemistry (IHC) markers have been introduced to assist the diagno- sis of $\mathrm{HD}(1,5)$. AchE has different disadvantages because it needs fresh frozen tissue, and also there are high rates of interobserver disagreement, and high false positive and false negative results (4). Several antigens have been assessed for IHC, but none of these markers overcome the problems of the diagnosis of HD (5). Recently calretinin has been introduced as a useful marker for the diagnosis of HD. Calretinin is a vitamin D dependent calcium binding protein involved in calcium signaling. Lack of its immunostaining in the nerve fibers and ganglion cells of colonic wall has been claimed to be characteristic of HD, but so far very few studies have evaluated the usefulness of calretinin with controversial results (3).

\section{Objectives}

Due to limited number of studies on this important subject, we aimed to investigate and describe calretinin immunostaining patterns in aganglionic segment of HD colon specimens and compare it with normoganglionic 
segment and control non-Hirschsprung's disease (NHD) patients to determine the diagnostic value of this new method.

\section{Patients and Methods}

A prospective study was conducted in 2.5 -year period, from 2011-2013 in Namazi hospital affiliated to Shiraz University of Medical Sciences. All the patients that underwent segmental resection of colon due to constipation and suspicion to HD were collected. Our gold standard test to diagnose HD was absence of ganglion cell in serial $\mathrm{H}$ and $\mathrm{E}$ stained sections from colectomy specimens. The resected specimens of the colon obtained were sampled full wall thickness at $2 \mathrm{~cm}$ intervals sequentially from the dentate line proximally. The distal and proximal margins were separately sampled totally. Then the sections were stained with $\mathrm{H}$ and $\mathrm{E}$. These $\mathrm{H}$ and $\mathrm{E}$ slides were evaluated carefully for the presence or lack of ganglion cells and nerve hypertrophy. The patients with lack of ganglion cells in the distal segment of colon were classified as HD group and the others with presence of ganglion cells through the whole length of specimen were classified as NHD. In total $27 \mathrm{HD}$ patients and 28 NHD patients were collected in the study period. Patients with inadequate clinical information were excluded.

Among the paraffin embedded blocks of HD patients a good full wall thickness section from aganglionic segment and one from normoganglionic segment were selected (but in $4 \mathrm{HD}$ patients due to total aganglionosis both of the blocks were selected from aganglionic area) and among the blocks of NHD patients one random block was selected. In total, 82 paraffin blocks (31 aganglionic and 51 normoganglionic) were examined which included blocks from aganglionic and normoganglionic zones of HD patients and blocks from normoganglionic NHD patients.

Calretinin staining was performed and IHC slides were seen without knowledge of the final diagnosis by two pathologists (Pathologist 1: experienced in diagnosing HD and Pathologist 2: inexperienced). Calretinin immunostaining was scored as either positive or negative. The location of staining (Nerve fibers of lamina propria and submucosa or ganglion cells of submucosa and muscularis propria) was also recorded.

Calretinin was considered as positive if any of the specific findings below were present: 1 ) granular staining of nerve fibers in the lamina propria and submucosa, 2) diffuse intense cytoplasmic or nuclear staining of ganglion cells in the myenteric or meissner plexuses.

Data analysis was done by SPSS 11.5 for Windows. We applied Cohen's kappa coefficient (with statistically significant level of $\mathrm{P}<0.05$ ) for determining the agreement between the calretinin IHC and the gold standard and between the decisions made by two pathologists. The diagnostic values (sensitivity, specificity, positive predictive value and negative predictive value) of calretinin IHC for both pathologists were calculated.

\section{Results}

In our prospective study we collected 27 patients with confirmed HD whose mean age varied from 2 days to 10 years and male to female ratio was 5.75 (Table 1). Of these patients 17 (63\%) were classic form of HD, 4 (15\%) long segment form and 6 (22\%) total colonic aganglionosis. Twenty one (77.8\%) patients presented in the first month of life, $2(7.4 \%)$ patients between 1 month and 1 year and 4 (14.8\%) after first year of age. Most common presenting symptoms were delayed meconium passing, chronic constipation, abdominal distention and vomiting. Also we collected 28 patients who underwent segmental colectomy, but serial $\mathrm{H}$ and $\mathrm{E}$ stained sections confirmed the presence of ganglion cells in whole length of the specimen. The age and gender of these patients are shown in Table 1. The causes of colectomy in these patients were chronic constipation (clinically suspicious to HD).

The analysis of serial $\mathrm{H}$ and $\mathrm{E}$ stained sections of the HD patients showed the absence of ganglion cells of submucosal and muscularis propria nerve plexuses in the distal part (aganglionic segment). The same evaluation of the NHD patients and normoganglionic segment of HD patients showed presence of ganglion cells mostly in the muscularis propria layer and less in the submucosa. Calretinin IHC slides were evaluated blindly by two pathologists independently. In this study typical calretinin immunostaining in normoganglionic sections was composed of granular staining of nerve fibers in lamina propria and submucosa and also intense cytoplasmic and nuclear staining of ganglion cells in the myenteric and Meissner plexuses (Figures 1 - 3). Negative staining of both nerve fibers and ganglion cells in all layers was considered as negative IHC (Figures 4 - 6). The results are shown in Tables 2 - 4.

According to pathologist 1 calretinin immunoexpression (nerve fiber or ganglion cell) was negative in all the layers (lamina propria, submucosa and muscularis propria) in 29 (92.6\%) sections. And according to pathologist 2 calretinin immunoexpression (nerve fiber or ganglion cell) was negative in all the layers in $27(87 \%)$ sections. Both pathologists have diagnosed case 25 as NHD (normoganglionosis). And both have reported nerve fiber immunoreactivity in muscularis propria of case 18 but final diagnoses were HD (aganglionosis). The cases No. 23 and 27 have been diagnosed as NHD by pathologist 2 due to focal staining of lamina propria nerve fibers but after reviewing of the slides the final consensus was aganglionosis by both pathologists.

The detailed results are shown in Tables $3-5$. According to both pathologists final diagnoses of all the sections from normoganglionic segment of HD patients were correct. Both pathologists have reported no immunoreactivity in lamina propria of cases No. 1, 15, 16 and 24, and pathologist 2 has also reported no immunoreactivity in lamina propria of case 12 but after reviewing of the slide the final consensus was immunoreactivity by both pathologists. 


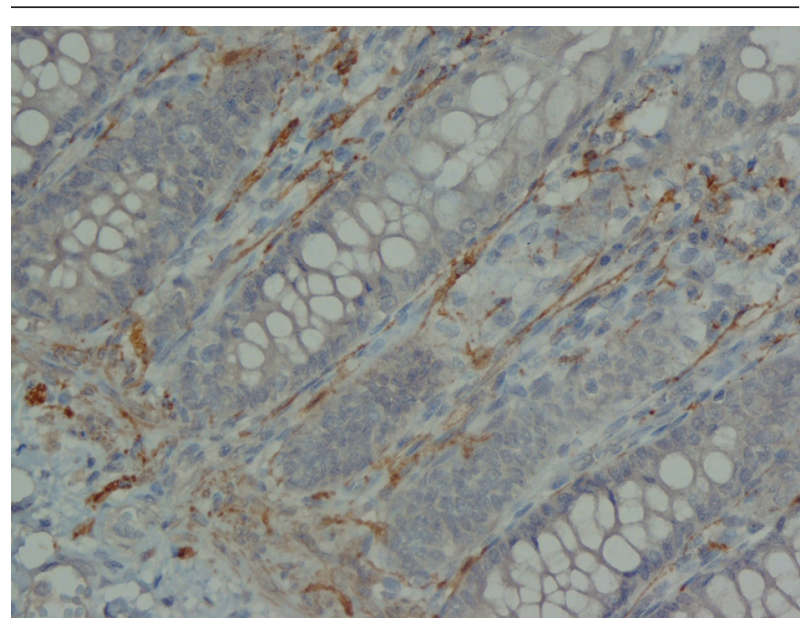

Figure 1. Low Power View of Full Wall Thickness Section Shows Calretinin Immunoreactivity in Lamina Propria, Submucosa and Muscularis Propria $(\times 100)$

Table 1. Demographic Findings of the Patients in the Study Group

\begin{tabular}{lcc}
\hline Variables & $\begin{array}{c}\text { Hirschsprung } \\
\text { Disease }\end{array}$ & $\begin{array}{c}\text { Non-Hirschsprung } \\
\text { Disease }\end{array}$ \\
\hline Number of Cases & 27 & 28 \\
Age, month ${ }^{\text {a }}$ & $21.3 \pm 28.1$ & $27 \pm 33.2$ \\
Gender $^{\text {b }}$ & & \\
Male & $23(85)$ & $21(75)$ \\
Female ${ }^{b}$ & $4(15)$ & $7(25)$ \\
\hline $\begin{array}{l}\text { a Mean } \pm \text { SD. } \\
\text { b No. } \%) .\end{array}$
\end{tabular}

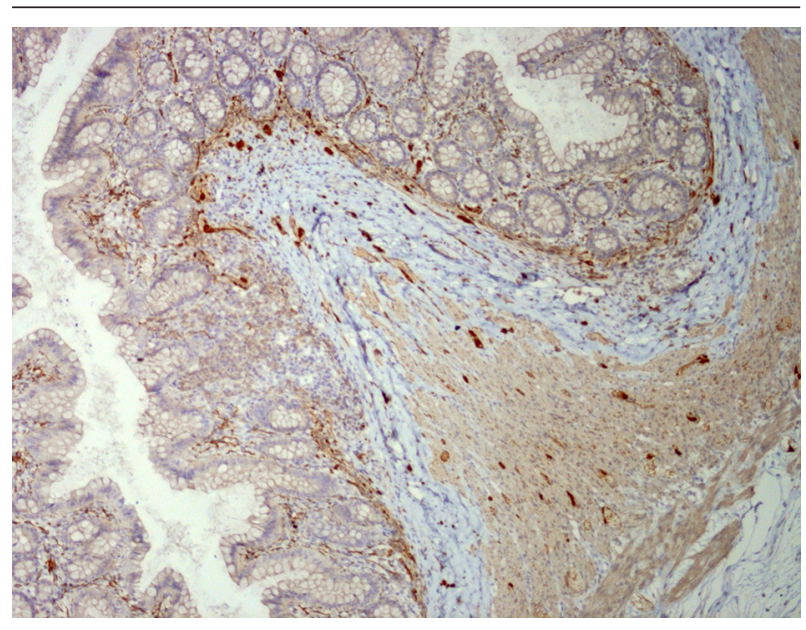

Figure 2. Lamina Propria Shows Calretinin Immunoreactivity in Nerve Fibers $(\times 250)$

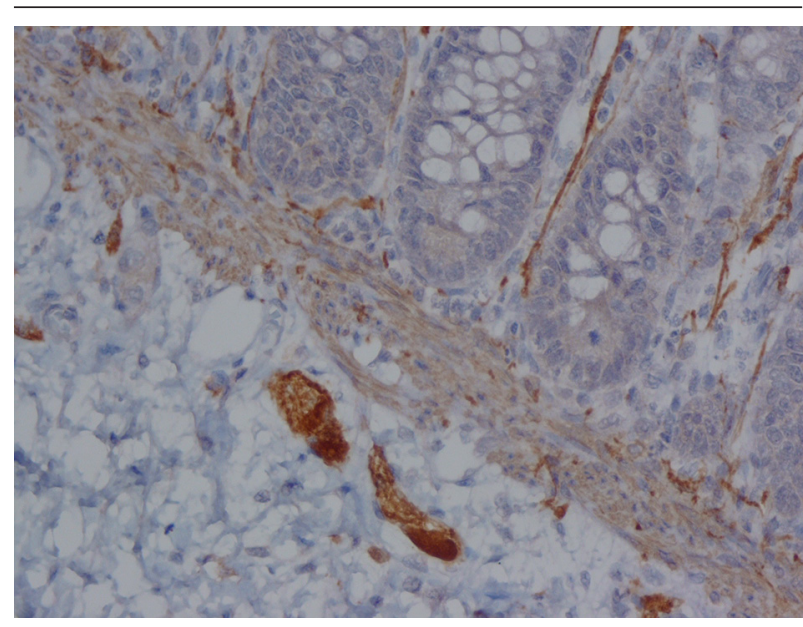

Figure 3. Strong Cytoplasmic and Nuclear Calretinin Immunoreactivity in Ganglion Cells of Submucosa $(\times 250)$

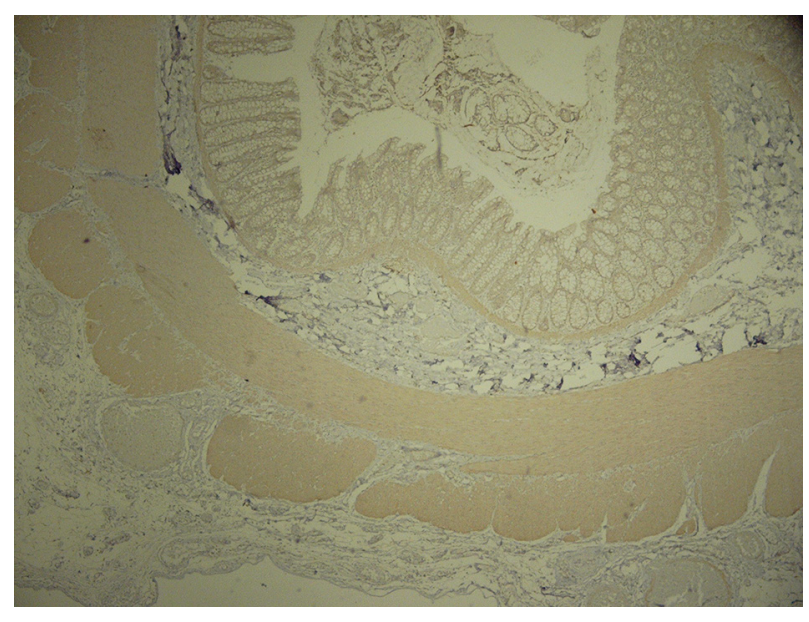

Figure 4. Low Power View of Full Wall Thickness Section Shows no Calretinin Immunoreactivity in Lamina Propria, Submucosa and Muscularis Propria $(\times 40)$

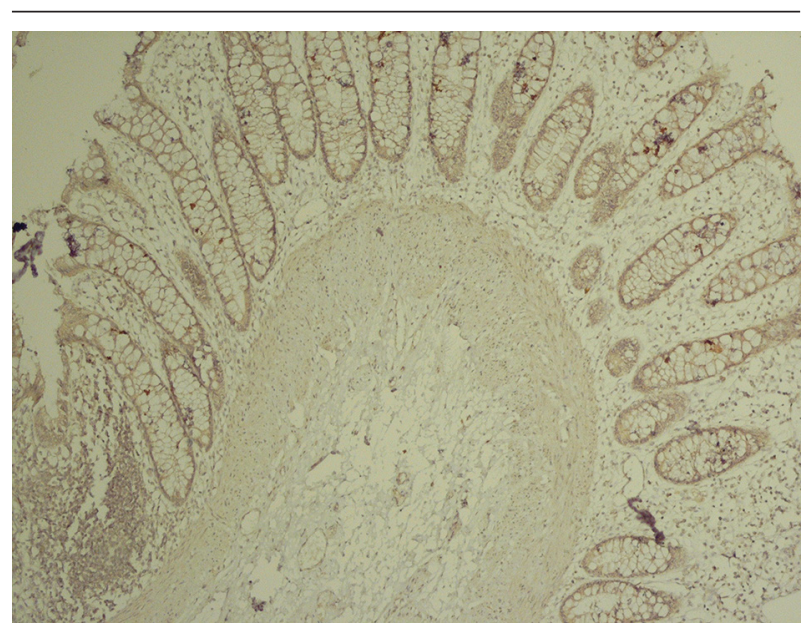

Figure 5. Submucosa Shows no Calretinin Immunoreactivity in Nerve Fibers and Ganglion Cells $(\times 100)$ 


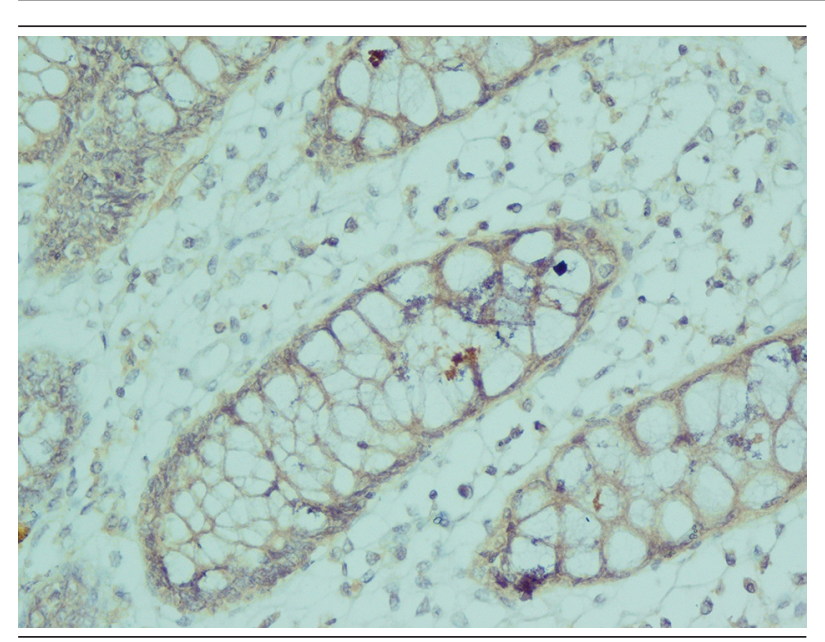

Figure 6. Lamina Propria Shows no Calretinin Immunoreactivity in Nerve Fibers $(\times 400)$

The diagnostic values (sensitivity, specificity, positive predictive value and negative predictive value) of calretinin IHC for both pathologists according to final diagnosis on each section, decision only on lamina propria and decision only on submucosa were calculated and shown in Table 6. The final diagnoses on calretinin IHC were compared with the gold standard, pathologist 1 diagnosed all the aganglionic sections correctly with only one false negative. The concordance was statistically perfect $(k=0.97 \pm 0.02, P<0.001)$. The comparison between decision only on lamina propria and gold standard showed perfect concordance $(\mathrm{k}=0.82 \pm 0.06, \mathrm{P}<0.001)$, and also by decision only on submucosa and gold standard showed perfect concordance. $(\mathrm{k}=1, \mathrm{P}<0.001)$.

The concordance between the final diagnoses of pathologist 2 and gold standard was also perfect. ( $\mathrm{k}=0.92$ $\pm 0.04, \mathrm{P}<0.001)$. Pathologist 2 failed to diagnose three aganglionic sections ( 3 false negatives) with no false positive. The comparison between decision only on lamina propria and gold standard showed substantial concordance $(\mathrm{k}=0.77 \pm 0.07, \mathrm{P}<0.001)$, and between decision only on submucosa and gold standard showed perfect concordance $(\mathrm{k}=1, \mathrm{P}<0.001)$.

The agreement in final diagnosis on calretinin IHC between the two pathologists was perfect $(\mathrm{k}=0.94 \pm 0.03, \mathrm{P}$ $<0.001$ ), for decision only on lamina propria was perfect $(\mathrm{k}=0.90 \pm 0.04, \mathrm{P}<0.001)$ and also for decision only on submucosa was perfect. $(\mathrm{k}=1, \mathrm{P}<0.001)$. The distribution of sections based on final diagnosis of each pathologist is shown in Tables 7 and 8.

\begin{tabular}{|c|c|c|c|c|c|c|c|c|c|}
\hline \multirow[t]{2}{*}{ Case No. } & \multirow[t]{2}{*}{ Type } & \multicolumn{4}{|c|}{ Pathologist 1} & \multicolumn{4}{|c|}{ Pathologist 2} \\
\hline & & LP & SM & MP & Final Dx & $\mathbf{L P}$ & SM & MP & Final Dx \\
\hline 1 & Classic & $\mathrm{N}$ & $\mathrm{N}$ & $\mathrm{N}$ & HD & $\mathrm{N}$ & $\mathrm{N}$ & $\mathrm{N}$ & HD \\
\hline 2 & Long & $\mathrm{N}$ & $\mathrm{N}$ & $\mathrm{N}$ & HD & $\mathrm{N}$ & $\mathrm{N}$ & $\mathrm{N}$ & HD \\
\hline 3 & Classic & $\mathrm{N}$ & $\mathrm{N}$ & $\mathrm{N}$ & HD & $\mathrm{N}$ & $\mathrm{N}$ & $\mathrm{N}$ & HD \\
\hline 4 & Classic & $\mathrm{N}$ & $\mathrm{N}$ & $\mathrm{N}$ & HD & $\mathrm{N}$ & $\mathrm{N}$ & $\mathrm{N}$ & HD \\
\hline 5 & Classic & $\mathrm{N}$ & $\mathrm{N}$ & $\mathrm{N}$ & HD & $\mathrm{N}$ & $\mathrm{N}$ & $\mathrm{N}$ & HD \\
\hline 6 & Classic & $\mathrm{N}$ & $\mathrm{N}$ & $\mathrm{N}$ & HD & $\mathrm{N}$ & $\mathrm{N}$ & $\mathrm{N}$ & HD \\
\hline 7 & Classic & $\mathrm{N}$ & $\mathrm{N}$ & $\mathrm{N}$ & HD & $\mathrm{N}$ & $\mathrm{N}$ & $\mathrm{N}$ & HD \\
\hline 8 & Classic & $\mathrm{N}$ & $\mathrm{N}$ & $\mathrm{N}$ & HD & $\mathrm{N}$ & $\mathrm{N}$ & $\mathrm{N}$ & HD \\
\hline 9 & Classic & $\mathrm{N}$ & $\mathrm{N}$ & $\mathrm{N}$ & HD & $\mathrm{N}$ & $\mathrm{N}$ & $\mathrm{N}$ & HD \\
\hline 10 & Classic & $\mathrm{N}$ & $\mathrm{N}$ & $\mathrm{N}$ & HD & $\mathrm{N}$ & $\mathrm{N}$ & $\mathrm{N}$ & HD \\
\hline 11 & Long & $\mathrm{N}$ & $\mathrm{N}$ & $\mathrm{N}$ & HD & $\mathrm{N}$ & $\mathrm{N}$ & $\mathrm{N}$ & HD \\
\hline $12^{b}$ & TCA & & & & & & & & \\
\hline (A) & & $\mathrm{N}$ & $\mathrm{N}$ & $\mathrm{N}$ & HD & $\mathrm{N}$ & $\mathrm{N}$ & $\mathrm{N}$ & HD \\
\hline (B) & & $\mathrm{N}$ & $\mathrm{N}$ & $\mathrm{N}$ & HD & $\mathrm{N}$ & $\mathrm{N}$ & $\mathrm{N}$ & HD \\
\hline 13 & Classic & $\mathrm{N}$ & $\mathrm{N}$ & $\mathrm{N}$ & HD & $\mathrm{N}$ & $\mathrm{N}$ & $\mathrm{N}$ & HD \\
\hline 14 & Long & $\mathrm{N}$ & $\mathrm{N}$ & $\mathrm{N}$ & HD & $\mathrm{N}$ & $\mathrm{N}$ & $\mathrm{N}$ & HD \\
\hline 15 & Long & $\mathrm{N}$ & $\mathrm{N}$ & $\mathrm{N}$ & HD & $\mathrm{N}$ & $\mathrm{N}$ & $\mathrm{N}$ & HD \\
\hline 16 & Classic & $\mathrm{N}$ & $\mathrm{N}$ & $\mathrm{N}$ & HD & $\mathrm{N}$ & $\mathrm{N}$ & $\mathrm{N}$ & HD \\
\hline 17 & Classic & $\mathrm{N}$ & $\mathrm{N}$ & $\mathrm{N}$ & HD & $\mathrm{N}$ & $\mathrm{N}$ & $\mathrm{N}$ & HD \\
\hline 18 & Classic & $\mathrm{N}$ & $\mathrm{N}$ & $\mathrm{P}$ & HD & $\mathrm{N}$ & $\mathrm{N}$ & $\mathrm{P}$ & HD \\
\hline 19 & Classic & $\mathrm{N}$ & $\mathrm{N}$ & $\mathrm{N}$ & HD & $\mathrm{N}$ & $\mathrm{N}$ & $\mathrm{N}$ & HD \\
\hline 20 & Classic & $\mathrm{N}$ & $\mathrm{N}$ & $\mathrm{N}$ & HD & $\mathrm{N}$ & $\mathrm{N}$ & $\mathrm{N}$ & HD \\
\hline 21 & Classic & $\mathrm{N}$ & $\mathrm{N}$ & $\mathrm{N}$ & HD & $\mathrm{N}$ & $\mathrm{N}$ & $\mathrm{N}$ & HD \\
\hline
\end{tabular}




\begin{tabular}{|c|c|c|c|c|c|c|c|c|c|}
\hline $22^{b}$ & TCA & & & & & & & & \\
\hline (A) & & $\mathrm{N}$ & $\mathrm{N}$ & $\mathrm{N}$ & HD & $\mathrm{N}$ & $\mathrm{N}$ & $\mathrm{N}$ & HD \\
\hline (B) & & $\mathrm{N}$ & $\mathrm{N}$ & $\mathrm{N}$ & HD & $\mathrm{N}$ & $\mathrm{N}$ & $\mathrm{N}$ & HD \\
\hline 23 & TCA & $\mathrm{N}$ & $\mathrm{N}$ & $\mathrm{N}$ & HD & $\mathrm{P}$ & $\mathrm{N}$ & $\mathrm{N}$ & NHD \\
\hline 24 & Classic & $\mathrm{N}$ & $\mathrm{N}$ & $\mathrm{N}$ & HD & $\mathrm{N}$ & $\mathrm{N}$ & $\mathrm{N}$ & HD \\
\hline 25 & TCA & $\mathrm{P}$ & $\mathrm{N}$ & $\mathrm{N}$ & NHD & $\mathrm{P}$ & $\mathrm{N}$ & $\mathrm{N}$ & NHD \\
\hline $26^{b}$ & TCA & & & & & & & & \\
\hline (A) & & $\mathrm{N}$ & $\mathrm{N}$ & $\mathrm{N}$ & HD & $\mathrm{N}$ & $\mathrm{N}$ & $\mathrm{N}$ & HD \\
\hline (B) & & $\mathrm{N}$ & $\mathrm{N}$ & $\mathrm{N}$ & HD & $\mathrm{N}$ & $\mathrm{N}$ & $\mathrm{N}$ & HD \\
\hline $27^{b}$ & TCA & & & & & & & & \\
\hline (A) & & $\mathrm{N}$ & $\mathrm{N}$ & $\mathrm{N}$ & HD & $\mathrm{P}$ & $\mathrm{N}$ & $\mathrm{N}$ & NHD \\
\hline (B) & & $\mathrm{N}$ & $\mathrm{N}$ & $\mathrm{N}$ & HD & $\mathrm{N}$ & $\mathrm{N}$ & $\mathrm{N}$ & HD \\
\hline \multicolumn{10}{|c|}{$\begin{array}{l}\text { a Abbreviations: Final Dx, final diagnosis; HD, Hirschsprung disease; LP, Lamina properia; MP, muscularis properia; N, negative; NHD, non-Hirschsprung } \\
\text { disease; P, positive; SM, submucosa; TCA, total colonic aganglionosis. } \\
\text { b These cases had two aganglionic sections. }\end{array}$} \\
\hline \multicolumn{10}{|c|}{$\begin{array}{l}\text { Table 3. Calretinin Immunoreactivity in Full Wall Thickness Sections From Hirschsprung Disease (HD) and Non-Hirschsprung Dis- } \\
\text { ease (NHD) Patients According to Pathologist } 1 \text { a }\end{array}$} \\
\hline \multirow{2}{*}{\multicolumn{2}{|c|}{ Location }} & \multicolumn{3}{|c|}{ Aganglionic Segment of HD } & \multicolumn{3}{|c|}{ Normoganglionic Segment of HD } & \multicolumn{2}{|c|}{ NHD } \\
\hline & & \multicolumn{2}{|c|}{ Positive } & Negative & \multicolumn{2}{|c|}{ Positive } & Negative & Positive & Negative \\
\hline \multicolumn{2}{|c|}{ Lamina propria } & \multicolumn{2}{|c|}{$1(3.3)$} & $30(96.7)$ & \multicolumn{2}{|c|}{$29(93.5)$} & $2(6.5)$ & $27(87.1)$ & $4(12.9)$ \\
\hline \multicolumn{2}{|c|}{ Submucosal nerve fibers } & \multicolumn{2}{|c|}{$0(0)$} & $31(100)$ & \multicolumn{2}{|c|}{$31(100)$} & $0(0)$ & $31(100)$ & $0(0)$ \\
\hline \multicolumn{2}{|c|}{ Submucosal ganglion cell } & \multicolumn{2}{|c|}{$0(0)$} & $31(100)$ & \multicolumn{2}{|c|}{$31(100)$} & $0(0)$ & $31(100)$ & $0(0)$ \\
\hline \multicolumn{2}{|c|}{ Muscularis propria } & \multicolumn{2}{|c|}{$1(3.3)$} & $30(96.7)$ & \multicolumn{2}{|c|}{$31(100)$} & $0(0)$ & $31(100)$ & $0(0)$ \\
\hline
\end{tabular}

a Data are presented as No. (\%).

Table 4. Calretinin Immunoreactivity in Full Wall Thickness Sections From Hirschsprung Disease (HD) and Non-Hirschsprung Disease (NHD) Patients According to Pathologist $2{ }^{\text {a }}$

\begin{tabular}{lcccccc}
\hline Location & \multicolumn{2}{c}{ Aganglionic Segment of HD } & Normoganglionic Segment of HD & \multicolumn{2}{c}{ NHD } \\
\cline { 2 - 7 } & Positive & Negative & Positive & Negative & Positive & Negative \\
\hline Lamina propria & $3(9.7)$ & $28(90.3)$ & $30(96.7)$ & $1(3.3)$ & $26(83.8)$ & $5(16.2)$ \\
Submucosal nerve fibers & $0(0)$ & $31(100)$ & $31(100)$ & $0(0)$ & $31(100)$ & $0(0)$ \\
Submucosal ganglion cell & $0(0)$ & $31(100)$ & $31(100)$ & $0(0)$ & $31(100)$ & $0(0)$ \\
Muscularis propria & $1(3.3)$ & $30(96.7)$ & $31(100)$ & $0(0)$ & $31(100)$ & $0(0)$ \\
\hline
\end{tabular}

a Data are presented as No. (\%).

Table 5. Calretinin Immunohistochemistry Results in Normoganglionic Segment of Hirschsprung Disease Patients a

\begin{tabular}{|c|c|c|c|c|c|c|c|c|c|}
\hline \multirow[t]{2}{*}{ Case No. ${ }^{b}$} & \multirow[t]{2}{*}{ Type } & \multicolumn{4}{|c|}{ Pathologist 1} & \multicolumn{4}{|c|}{ Pathologist 2} \\
\hline & & $\mathbf{L P}$ & SM & MP & Final Dx & LP & SM & MP & Final Dx \\
\hline 1 & Classic & $\mathrm{P}$ & $\mathrm{P}$ & $\mathrm{P}$ & NG & $P$ & $P$ & $\mathrm{P}$ & NG \\
\hline 2 & Long & $\mathrm{P}$ & $\mathrm{P}$ & $\mathrm{P}$ & NG & $\mathrm{P}$ & $\mathrm{P}$ & $\mathrm{P}$ & NG \\
\hline 3 & Classic & $P$ & $P$ & $\mathrm{P}$ & NG & $\mathrm{P}$ & $\mathrm{P}$ & $\mathrm{P}$ & NG \\
\hline 4 & Classic & $\mathrm{P}$ & $\mathrm{P}$ & $\mathrm{P}$ & NG & $\mathrm{P}$ & $\mathrm{P}$ & $\mathrm{P}$ & NG \\
\hline 5 & Classic & $\mathrm{P}$ & $\mathrm{P}$ & $P$ & NG & $\mathrm{P}$ & $\mathrm{P}$ & $P$ & NG \\
\hline 6 & Classic & $\mathrm{P}$ & $\mathrm{P}$ & $\mathrm{P}$ & NG & $\mathrm{P}$ & $\mathrm{P}$ & $\mathrm{P}$ & NG \\
\hline 7 & Classic & $\mathrm{N}$ & $\mathrm{P}$ & $P$ & NG & $\mathrm{P}$ & $\mathrm{P}$ & $P$ & NG \\
\hline 8 & Classic & $\mathrm{N}$ & $P$ & $\mathrm{P}$ & NG & $\mathrm{N}$ & $P$ & $\mathrm{P}$ & NG \\
\hline
\end{tabular}


Anbardar MH et al.

\begin{tabular}{|c|c|c|c|c|c|c|c|c|c|}
\hline 9 & Classic & $\mathrm{P}$ & $\mathrm{P}$ & $\mathrm{P}$ & NG & $\mathrm{P}$ & $\mathrm{P}$ & $\mathrm{P}$ & NG \\
\hline 10 & Classic & $\mathrm{P}$ & $\mathrm{P}$ & $\mathrm{P}$ & NG & $\mathrm{P}$ & $\mathrm{P}$ & $\mathrm{P}$ & NG \\
\hline 11 & Long & $\mathrm{P}$ & $\mathrm{P}$ & $\mathrm{P}$ & NG & $\mathrm{P}$ & $\mathrm{P}$ & $\mathrm{P}$ & NG \\
\hline 13 & Classic & $\mathrm{P}$ & $\mathrm{P}$ & $\mathrm{P}$ & NG & $P$ & $\mathrm{P}$ & $P$ & NG \\
\hline 14 & Long & $\mathrm{P}$ & $\mathrm{P}$ & $\mathrm{P}$ & NG & $\mathrm{P}$ & $\mathrm{P}$ & $P$ & NG \\
\hline 15 & Long & $\mathrm{P}$ & $\mathrm{P}$ & $\mathrm{P}$ & NG & $P$ & $\mathrm{P}$ & $P$ & NG \\
\hline 16 & Classic & $\mathrm{P}$ & $\mathrm{P}$ & $\mathrm{P}$ & NG & $\mathrm{P}$ & $\mathrm{P}$ & $\mathrm{P}$ & NG \\
\hline 17 & Classic & $\mathrm{P}$ & $\mathrm{P}$ & $\mathrm{P}$ & NG & $P$ & $\mathrm{P}$ & $P$ & NG \\
\hline 18 & Classic & $\mathrm{P}$ & $\mathrm{P}$ & $\mathrm{P}$ & NG & $\mathrm{P}$ & $\mathrm{P}$ & $\mathrm{P}$ & NG \\
\hline 19 & Classic & $\mathrm{P}$ & $\mathrm{P}$ & $\mathrm{P}$ & NG & $P$ & $\mathrm{P}$ & $P$ & NG \\
\hline 20 & Classic & $\mathrm{P}$ & $\mathrm{P}$ & $\mathrm{P}$ & NG & $P$ & $\mathrm{P}$ & $P$ & NG \\
\hline 21 & Classic & $\mathrm{P}$ & $\mathrm{P}$ & $\mathrm{P}$ & NG & $P$ & $\mathrm{P}$ & $P$ & NG \\
\hline 23 & TCA & $\mathrm{P}$ & $\mathrm{P}$ & $\mathrm{P}$ & NG & $\mathrm{P}$ & $\mathrm{P}$ & $\mathrm{P}$ & NG \\
\hline 24 & Classic & $\mathrm{P}$ & $\mathrm{P}$ & $\mathrm{P}$ & NG & $P$ & $\mathrm{P}$ & $P$ & NG \\
\hline 25 & TCA & $\mathrm{P}$ & $\mathrm{P}$ & $\mathrm{P}$ & NG & P & $\mathrm{P}$ & $\mathrm{P}$ & NG \\
\hline
\end{tabular}

a Abbreviations: Final Dx, final diagnosis; LP, lamina propria; MP, muscularis propria; N, negative; NG, normoganglionosis; SM, submucosa; P, positive; TCA, total colonic aganglionosis.

b Cases 12, 22, 26 and 27 had no normoaganglionic section.

\begin{tabular}{|c|c|c|c|c|c|c|c|c|}
\hline \multirow[t]{2}{*}{ Case No. } & \multicolumn{4}{|c|}{ Pathologist 1} & \multicolumn{4}{|c|}{ Pathologist 2} \\
\hline & LP & SM & MP & Final Dx & $\mathbf{L P}^{\mathrm{a}}$ & SM & MP & Final Dx \\
\hline 1 & $\mathrm{~N}$ & $\mathrm{P}$ & $\mathrm{P}$ & NHD & $\mathrm{N}$ & $\mathrm{P}$ & $\mathrm{P}$ & NHD \\
\hline 2 & $\mathrm{P}$ & $\mathrm{P}$ & $\mathrm{P}$ & NHD & $\mathrm{P}$ & $\mathrm{P}$ & $\mathrm{P}$ & NHD \\
\hline 3 & $\mathrm{P}$ & $\mathrm{P}$ & $P$ & NHD & $P$ & $\mathrm{P}$ & $P$ & NHD \\
\hline 4 & $\mathrm{P}$ & $\mathrm{P}$ & $\mathrm{P}$ & NHD & $\mathrm{P}$ & $\mathrm{P}$ & $P$ & NHD \\
\hline 5 & $\mathrm{P}$ & $\mathrm{P}$ & $\mathrm{P}$ & NHD & $\mathrm{P}$ & $\mathrm{P}$ & $\mathrm{P}$ & NHD \\
\hline 6 & $\mathrm{P}$ & $\mathrm{P}$ & $P$ & NHD & $\mathrm{P}$ & $\mathrm{P}$ & $P$ & NHD \\
\hline 7 & $\mathrm{P}$ & $\mathrm{P}$ & $\mathrm{P}$ & NHD & $\mathrm{P}$ & $\mathrm{P}$ & $\mathrm{P}$ & NHD \\
\hline 8 & $\mathrm{P}$ & $\mathrm{P}$ & $\mathrm{P}$ & NHD & $\mathrm{P}$ & $\mathrm{P}$ & $P$ & NHD \\
\hline 9 & $\mathrm{P}$ & $\mathrm{P}$ & $P$ & NHD & $P$ & $\mathrm{P}$ & $P$ & NHD \\
\hline 10 & $\mathrm{P}$ & $\mathrm{P}$ & $\mathrm{P}$ & NHD & $\mathrm{P}$ & $\mathrm{P}$ & $\mathrm{P}$ & NHD \\
\hline 11 & $\mathrm{P}$ & $\mathrm{P}$ & $P$ & NHD & $P$ & $\mathrm{P}$ & $P$ & NHD \\
\hline 12 & $\mathrm{P}$ & $\mathrm{P}$ & $\mathrm{P}$ & NHD & $\mathrm{N}$ & $\mathrm{P}$ & $P$ & NHD \\
\hline 13 & $\mathrm{P}$ & $\mathrm{P}$ & $P$ & NHD & $\mathrm{P}$ & $\mathrm{P}$ & $P$ & NHD \\
\hline 14 & $\mathrm{P}$ & $\mathrm{P}$ & $\mathrm{P}$ & NHD & $\mathrm{P}$ & $\mathrm{P}$ & $\mathrm{P}$ & NHD \\
\hline 15 & $\mathrm{~N}$ & $\mathrm{P}$ & $\mathrm{P}$ & NHD & $\mathrm{N}$ & $\mathrm{P}$ & $P$ & NHD \\
\hline 16 & $\mathrm{~N}$ & $\mathrm{P}$ & $\mathrm{P}$ & NHD & $\mathrm{N}$ & $\mathrm{P}$ & $P$ & NHD \\
\hline 17 & $\mathrm{P}$ & $\mathrm{P}$ & $P$ & NHD & $P$ & $\mathrm{P}$ & $P$ & NHD \\
\hline 18 & $\mathrm{P}$ & $\mathrm{P}$ & $\mathrm{P}$ & NHD & $\mathrm{P}$ & $\mathrm{P}$ & $\mathrm{P}$ & NHD \\
\hline 19 & $P$ & $\mathrm{P}$ & $P$ & NHD & $P$ & $\mathrm{P}$ & $P$ & NHD \\
\hline 20 & $\mathrm{P}$ & $\mathrm{P}$ & $\mathrm{P}$ & NHD & $\mathrm{P}$ & $\mathrm{P}$ & $\mathrm{P}$ & NHD \\
\hline 21 & $P$ & $\mathrm{P}$ & $P$ & NHD & $P$ & $\mathrm{P}$ & $P$ & NHD \\
\hline 22 & $\mathrm{P}$ & $\mathrm{P}$ & $\mathrm{P}$ & NHD & $\mathrm{P}$ & $\mathrm{P}$ & $\mathrm{P}$ & NHD \\
\hline 23 & $P$ & $\mathrm{P}$ & $P$ & NHD & $P$ & $\mathrm{P}$ & $P$ & NHD \\
\hline 24 & $\mathrm{~N}$ & $\mathrm{P}$ & $\mathrm{P}$ & NHD & $\mathrm{N}$ & $\mathrm{P}$ & $\mathrm{P}$ & NHD \\
\hline 25 & $P$ & $\mathrm{P}$ & $P$ & NHD & $P$ & $\mathrm{P}$ & $P$ & NHD \\
\hline
\end{tabular}


Anbardar MH et al.

\begin{tabular}{lllllllll}
\hline $\mathbf{2 6}$ & P & P & P & NHD & P & P & P & NHD \\
$\mathbf{2 7}$ & P & P & P & NHD & P & P & P & NHD \\
$\mathbf{2 8}$ & P & P & P & NHD & P & P & P & NHD \\
\hline
\end{tabular}

${ }^{a}$ Abbreviations: Final Dx, final diagnosis; HD, Hirschsprung disease; LP, lamina propria; MP, muscularis propria; N, negative; NHD, non-Hirschsprung disease; P, positive; SM, submucosa.

Table 7. Statistical Evaluation of Calretinin Immunohistochemistry According to Both Pathologists' Final Diagnosis, Decision on Lamina Propria and Submucosa Compare With Gold Standard ${ }^{\text {a }}$

\begin{tabular}{|c|c|c|c|c|c|c|}
\hline Pathologist & Kappa & PValue & Sensitivity ${ }^{\mathrm{b}}$ & Specificity $^{\mathrm{b}}$ & $\mathbf{P P V}^{\mathrm{b}}$ & $\mathbf{N P V}^{\mathrm{b}}$ \\
\hline \multicolumn{7}{|c|}{ Final Diagnosis } \\
\hline 1 & 0.97 & $<0.001$ & 96.7 & 100 & 100 & 98 \\
\hline 2 & 0.92 & $<0.001$ & 90.3 & 100 & 100 & 94.4 \\
\hline \multicolumn{7}{|c|}{ Lamina propria } \\
\hline 1 & 0.82 & $<0.001$ & 96.7 & 88.2 & 83.3 & 97.8 \\
\hline 2 & 0.77 & $<0.001$ & 90.3 & 88.2 & 82.3 & 93.7 \\
\hline \multicolumn{7}{|l|}{ Submucosa } \\
\hline 1 & 1 & $<0.001$ & 100 & 100 & 100 & 100 \\
\hline 2 & 1 & $<0.001$ & 100 & 100 & 100 & 100 \\
\hline
\end{tabular}

a Abbreviations: Kappa, concordance; NPV, negative predictive value; PPV, positive predictive value.

b Data are presented as \%.

Table 8. Distribution of Sections According to Pathologists' Opinion ${ }^{a}$

\begin{tabular}{lcc}
\hline Pathologist 1 & Pathologist 2 & Number/Total, \% \\
\hline Aganglionosis (HD) & Aganglionosis (HD) & $28.82(34.1)$ \\
Aganglionosis (HD) & Normoganglionosis (NonHD) & $2.82(2.5)$ \\
Normoganglionosis (NonHD) & Aganglionosis (HD) & $0.82(0)$ \\
Normoganglionosis (NonHD) & Normoganglionosis (NonHD) & $52.82(63.4)$ \\
\hline
\end{tabular}

a Abbreviations: HD, Hirschsprung's disease.

\section{Discussion}

HD is a common congenital intestinal disorder with absence of ganglion cells in the colonic wall. The diagnosis is based on clinical data, imaging studies and histological features together. However, rectal muscle biopsy (stained by $\mathrm{H}$ and $\mathrm{E}$ stain) is the most common procedure for the diagnosis of $\mathrm{HD}(1)$.

There are diagnostic pitfalls of H AND E stained sections, so a number of ancillary methods such as acetyl cholinesterase (AchE) and immunohistochemistry (IHC) have been introduced to assist the diagnosis of HD, however until now there is no definite method for the diagnosis of HD except for absence of ganglion cells in the rectal muscle wall in $\mathrm{H}$ and $\mathrm{E}$ sections, and overall there is no consensus for using the ancillary methods and markers by immunohistochemistry (4). Recently calretinin immunostaining of rectal biopsies has been introduced by a few authors (4-10).

IHC for calretinin was first proposed by Barshack et al. in HD patients to increase the diagnostic accuracy of $\mathrm{H}$ AND E stain (6). The results of a few previous studies have suggested that calretinin IHC is a useful ancillary meth- od in the pathological evaluation of sections from HD patients (7) with higher diagnostic values compared to H AND E stained sections (8). According to other studies, the calretinin IHC is superior to AchE for the diagnosis or exclusion of $\mathrm{HD}$ (7-10).

The results of our study showed several advantages for calretinin. Calretinin immunostaining is superior to AchE because it can be stained on formalin fixed paraffin embedded tissue, but AchE should be stained on fresh frozen tissue (4). Also calretinin is available in every pathology department with IHC lab, needs less experience, and is easy to interpret. It can also be performed on paraffin embedded formalin-fixed sections of archival specimens (7-11).

In our study, calretinin immunoreactivity was present in both nerve fibers and ganglion cells. Immunoreactive nerve fibers showed variable intensity, granular and fibrillar cytoplasmic staining in the lamina propria, submucosa and muscularis propria. In the lamina propria they present as very thin elongated fibrils that surround the glands and in most of the normoganglionic segments 
they show diffuse staining (Figure 2). In the submucosa they present thicker and shorter fibrils and there were no difference between superficial and deep submucosa. (Figure 1). Ganglion cells mostly showed intense granular cytoplasmic and nuclear staining both in submucosa and muscularis propria (Figure 3 ).

According to our study, calretinin is also a good marker for ganglion cells. We recommend the use of calretinin in posterior myectomy specimens to find ganglion cells. Kacar et al. suggested that continuous nerve staining in the lamina propria, muscularis mucosa and submucosa would help to overcome problems with small biopsies (3). This continuous pattern was present in most normoganglionic segments.

Another benefit of calretinin immunostaining is the presence of positive control in the sections of the submucosa, i.e. some nonneuronal cells such as histiocytes and mast cells showed cytoplasmic and nuclear immunoreactivity that can be considered as internal positive control (Figure 7). In typical aganglionic segments, there was no immunoreactivity in nerve fibers or ganglion cells but the cytoplasm of mast cells or histiocytes showed weak to moderate staining (11) (Figure 7).

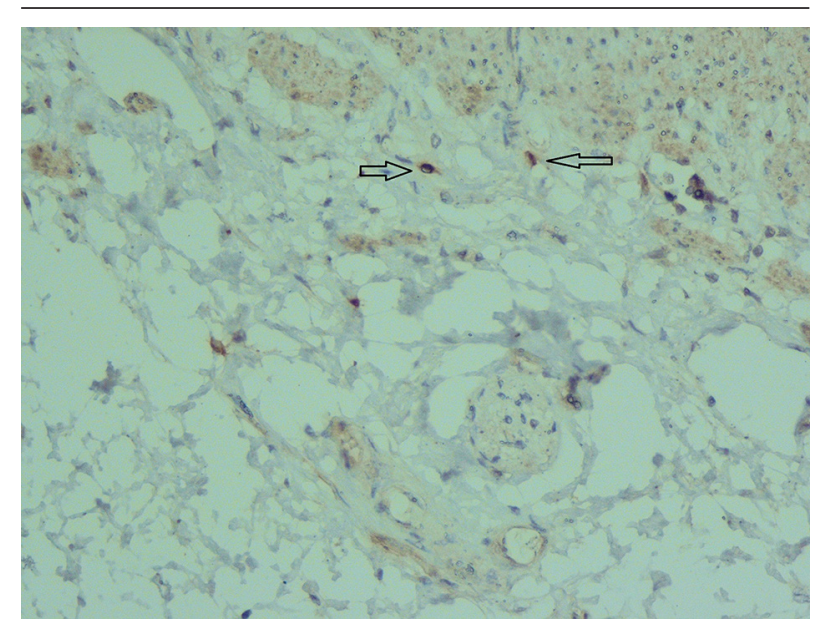

Figure 7. Submucosa Shows Two Mast Cells (Arrow) Which are Stained by Calretinin and can be Used as Positive Control $(\times 400)$

Kapur et al. suggested that immunoreactive nerve fibers should be completely lost in biopsies from aganglionic zone and presence of sparse immunoreactive fibers is associated with presence of ganglion cells (7). Also Holland et al. recommended that even focal and pale staining of nerve fibers is not seen in HD patients (12).

In our study both pathologists diagnosed aganglionic sections with perfect concordance compared with gold standard. These results are compatible with previous studies $(4,6,8,9,12-14)$. The diagnostic values of calretinin IHC for diagnosing aganglionic sections of HD patients were great with $100 \%$ specificity and positive predictive value. There was no false positive result. So calretinin IHC can be a useful tool in the diagnosis of HD.
Several previous reports also have noted the possibility of false negative results in calretinin IHC as in our study, due to immunostaining in some nonneuronal cells $(6,7$, $9,12)$. In our study the decisions of both pathologists on only submucosa showed perfect concordance with gold standard and there was no false positive or false negative result. This shows the importance of attention to submucosa in interpretation of calretinin IHC results. There was no difference between superficial and deep submucosa or between nerve fibers and ganglion cells. However, some of the previous studies also showed loss of immunoreactivity of nerve fibers and ganglion cells in submucosa $(4,6,8,9,12-14)$. Some of them reported large nerves in the submucosa with immunoreactive axons that are different from positive nerve fibers in superficial submu$\operatorname{cosa}(7,12,13)$ but we did not find these fibers in our cases. Guinard-Samuel et al. believed that this positive staining of some nerve fibers could indicate the beginning of transitional zone (9).

The decisions of both pathologists on only lamina properia showed good concordance with gold standard. There were six false positive results and one to three false negative cases. In some of previous studies, the calretinin IHC in lamina propria showed high concordance $(4,11,13$, 14). According to our calretinin results on both lamina propria and submucosa together, there is a perfect concordance with gold standard and the result of each component must be interpreted in association with other components.

In our study, the concordance between calretinin IHC and gold standard was also perfect for inexperienced pathologists to diagnose aganglionosis. The same results have also been reported previously by some authors (1517). Guinard-Samuel et al. noted that interpretation of AchE histochemistry is much more difficult for an inexperienced pathologist compared with calretinin IHC (9).

We had six cases of total colonic aganglionosis, one of them showed focal immunoreactivity of nerve fibers in lamina propria and was diagnosed as non HD in calretinin immunostaining by both pathologists. In previous study on total colonic aganglionosis conducted by Morris et al. they concluded that calretinin staining is superior to AchE especially in rectal biopsies from these patients (10).

In one of our HD patients, faint positive nerve fiber staining is seen in the muscularis propria. Hiradfar et al. also reported this finding in two cases (13). This could be due to the beginning of transitional zone. In conclusion, our results demonstrated that calretinin IHC is a very useful, valuable, sensitive and specific technique for detecting aganglionosis in patients suspicious to HD. Loss of calretinin immunostaining in lamina propria and especially submucosa is characteristic of HD. The use of this method on different specimens of these patients can help for accurate diagnosis, reduce the need for repeat biopsy and serial sectioning of blocks. Interpretation of calretinin IHC is easy and simple and does not need es- 
pecial experience but must be interpreted carefully in association with $\mathrm{H}$ and $\mathrm{E}$ stained sections and clinical data.

\section{References}

1. Immunohistochemical study of enteric nervous system in hirschsprung's disease and intestinal neuronal dysplasia. Geramizadeh B, Akbarzadeh E, Izadi B, Foroutan HR, Heidari T. Histol Histopathol. 2013;28(3):345.

2. Practical pathology and genetics of Hirschsprung's disease. Kapur RP. Semin Pediatr Surg. 2009;18(4):212.

3. Calretinin immunohistochemistry in Hirschsprung's disease: An adjunct to formalin-based diagnosis. Kacar A, Arikok AT, Azili MN, Ekberli Agirbas G, Tiryaki T. Turk J Gastroenterol. 2012;23(3):226.

4. Can we stop looking? Immunohistochemistry and the diagnosis of Hirschsprung disease. Kapur RP. Am J Clin Pathol. 2006;126(1):9. doi:10.1309/T7RE-Y1N4-3FML-7AA8. [PubMed:16753604]

5. Hirschsprung's disease: the Australian Paediatric Surveillance Unit's experience. Singh SJ, Croaker GD, Manglick P, Wong CL, Athanasakos H, Elliott E, et al. Pediatr Surg Int. 2003;19(4):247.

6. The loss of calretinin expression indicates aganglionosis in Hirschsprung's disease. Barshack I, Fridman E, Goldberg I, Chowers Y, Kopolovic J. J Clin Pathol. 2004;57(7):712.

7. Calretinin immunohistochemistry versus acetylcholinesterase histochemistry in the evaluation of suction rectal biopsies for Hirschsprung Disease. Kapur RP, Reed RC, Finn LS, Patterson K, Johanson J, Rutledge JC. Pediatr Dev Pathol. 2009;12(1):6.

8. Hirschsprung disease, associated syndromes and genetics: a review. Amiel J, Sproat-Emison E, Garcia-Barcelo M, Lantieri F, Burzynski G, Borrego S, et al. J Med Genet. 2008;45(1):1.
9. Calretinin immunohistochemistry: a simple and efficient tool to diagnose Hirschsprung disease. Guinard-Samuel V, Bonnard A, De Lagausie P, Philippe-Chomette P, Alberti C, El Ghoneimi A, et al. Mod Pathol. 2009;22(10):1379.

10. A study of calretinin in Hirschsprung pathology, particularly in total colonic aganglionosis. Morris MI, Soglio DB, Ouimet A, Aspirot A, Patey N. J Pediatr Surg. 2013;48(5):1037.

11. Diagnostic tests in Hirschsprung disease: a systematic review. de Lorijn F, Kremer LC, Reitsma JB, Benninga MA. J Pediatr Gastroenterol Nutr. 2006;42(5):496

12. Calretinin immunostaining as an adjunct in the diagnosis of Hirschsprung disease. Holland SK, Ramalingam P, Podolsky RH, Reid-Nicholson MD, Lee JR. Ann Diagn Pathol. 2011;15(5):323.

13. Calretinin Immunohistochemistery: An Aid in the Diagnosis of Hirschsprung's Disease. Hiradfar M, Sharifi N, Khajedaluee M, Zabolinejad N, Taraz Jamshidi S. Iran J Basic Med Sci. 2012;15(5):1053.

14. A useful panel for the diagnosis of Hirschsprung disease in rectal biopsies: calretinin immunostaining and acetylcholinesterase histochesmistry. de Arruda Lourencao PL, Takegawa BK, Ortolan EV, Terra SA, Rodrigues MA. Ann Diagn Pathol. 2013;17(4):352.

15. Hirschsprung disease and use of calretinin in inadequate rectal suction biopsies. Gonzalo DH, Plesec T. Arch Pathol Lab Med. 2013;137(8):1099.

16. Calretinin immunohistochemistry: A new cost-effective and easy method for diagnosis of Hirschsprung's disease. Kannaiyan L, Madabhushi S, Malleboyina R, Are NK, Reddy KR, Rao B. J Indian Assoc Pediatr Surg. 2013;18(2):66.

17. Hirschsprung disease. Evaluation of calretinin and S-100 as ancillary methods for the diagnosis of aganglionosis in rectal biopsies. De la Torre L, Santos K. Acta Pediatr Mex. 2012;33(5):246. 